\title{
Stringent and result-oriented training requirements at the heart of research funding opportunities: the case of the CSA FAST funding activity and the HiClBaS project
}

Simon Thibault, Guillaume Allain, Olivier Côté, Mireille Ouellet, Deven Patel, et al.

Simon Thibault, Guillaume Allain, Olivier Côté, Mireille Ouellet, Deven Patel, Cédric Vallée, Denis Brousseau, Anne-Sophie Poulin-Girard, "Stringent and result-oriented training requirements at the heart of research funding opportunities: the case of the CSA FAST funding activity and the HiCIBaS project," Proc. SPIE 11143, Fifteenth Conference on Education and Training in Optics and Photonics: ETOP 2019, 1114304 (2 July 2019); doi:

$10.1117 / 12.2523855$ 


\title{
Stringent and result-oriented training requirements at the heart of research funding opportunities: the case of the CSA FAST funding activity and the HiCIBaS project
}

Simon Thibault ${ }^{1}$, Guillaume Allain, Olivier Côté, Mireille Ouellet, Deven Patel, Cédric Vallée, Denis Brousseau, Anne-Sophie Poulin-Girard

Centre for optics, photonics and laser, Université Laval, 2375 rue de la Terrasse, Québec Canada G1V 0A6

\begin{abstract}
Funding opportunities in science are essential to the research and development ecosystem. Numerous and competitive, the vast majority focus on scientific accomplishment. While the advancement of science remains a top priority, some funding agencies started to reshape their programs to include strict training requirements, from training plans included in proposals to regular evaluations of training progress. At the centre of this change is the recognition of the universities and colleges educational mission through research, and the need for a highly qualified workforce serving industry, science, and research. It is this need for applied research training, expressed by the Canadian aerospace community, that led to the creation of the Canadian Space Agency's FAST (Flights and Fieldwork for the Advancement of Science and Technology) funding activity in 2011. Among the three main objectives of the 2017 opportunity, two target training the next and current generations of scientists and engineers for space-related areas in Canada by (1) developing and maintaining a critical mass of researchers, and (2) increasing the level of student employability by exposing them to practical experiences. In this paper, we report about the context behind CSA FAST's creation, the funding opportunity model, and the impact of the funding activity. Concrete results are also shown for the HiCIBaS project, funded by CSA FAST 2015, an ambitious balloon-borne mission with an optical payload for wavefront sensing and exoplanet imaging that was led by 5 graduate students as part of their master's program, and that culminated with a stratospheric balloon flight in August 2018.
\end{abstract}

Keywords: Practical training, Education, Funding opportunities, , Astronomical instrumentation, Optics and photonics, International collaboration, Soft skills

\section{CONTEXT: SPACE RESEARCH AND INDUSTRY IN CANADA}

Canada's involvement in space exploration is not new, from the launch of the Alouette satellite in 1962 to the participation in the NASA shuttle program that led to the rollout of Canadarm in 1981. Several Canadian astronauts participated in space missions, including Chris Hadfield, who commanded the International Space Station in 2013, and fascinated the planet with his interpretation of Space Oddity. The Canadian Space Agency (CSA) was established in 1989 to support the national space industry and provide government funding to bolster innovation in the field. Its mission to this day is to advance the knowledge of space and use its discoveries for the good of Canadians and of all humanity.

In 2010, CSA held a community-led workshop on suborbital platforms and nanosatellites that brought together more than 180 researchers, students and CSA managers, mostly from Canada, but also from Europe and the United States [1]. The 2010 Workshop on Suborbital platforms and nanosatellites was motivated by previous activities, including a CSA-led Nanosatellites Workshop (2004) and a meeting

${ }^{1}$ Simon.thibault@phy.ulaval.ca

Fifteenth Conference on Education and Training in Optics and Photonics: ETOP 2019, edited by

Anne-Sophie Poulin-Girard, Joseph A. Shaw, Proc. of SPIE Vol. 11143, 1114304 - (C) 2019

SPIE, ICO, IEEE, OSA · CCC code: 0277-786X/19/\$18 · doi: 10.1117/12.2523855 
of the user community for suborbital platforms in 2007. One of the key elements that came up from these meetings is the recognition of the value of graduate training. It is therefore not surprising that discussions on various types of suborbital platforms (aircrafts, balloons, sounding rockets) and nanosatellites in 2010 focused not only on research activities to further knowledge, but also on research training opportunities to train the next generation of scientists and engineers with skills in instrument design, data analysis and project management. The 2010 CSA workshop led to the creation of the CSA-FAST funding initiative the same year. Section 2 briefly describes the FAST funding activity, a research funding opportunity with strict training requirements, while section 3 depicts the HiCIBaS project, a Université Laval-based research project funded through the FAST funding activity in 2015. Section 4 presents the results of the training plan of HiCIBaS with quantitative and qualitative data gathered among HiCIBaS' participants. Section 5 highlights the efforts made to reach out to the community regarding the science and engineering behind $\mathrm{HiCIBaS}$.

\section{CSA-FAST FUNDING INITIATIVE: TRAINING TOMORROW'S WORKFORCE}

The FAST (Flights and Fieldwork for the Advancement of Science and Technology) funding initiative supports space research in post-secondary institutions in Canada [2]. The funding activity is centered around four goals: (1) developing and maintaining the expertise of the next generation of space researchers and other professionals in space-related fields in Canada, (2) providing students with opportunities to participate in hands-on activities to gain knowledge and acquire the skills sought by employers in the space sector, (3) increasing scientific knowledge, and (4) developing new technologies. Since the establishment of the funding activity in 2010, FAST has funded 78 projects to train over 600 participants including undergraduate students (44\% of all trainees), graduate students (42\%), and postdoctoral fellows (14\%).

The creation of this funding opportunity arose from a genuine preoccupation from the research and development community in the Canadian space sector. Its main particularity is the stringent and resultoriented training requirements accompanying research funding. As opposed to other sources of funding that require some sort of training intent, FAST really puts training at the centre of its funding activity. The students from the HiCIBaS project had the opportunity to experience this kind of training when the project received funding in 2015 to build a balloon-borne payload [3].

\section{HICIBAS PROJECT: ONE NIGHT ON A BALLOON}

HiCIBaS (High-Contrast Imaging Balloon System) is a balloon borne telescope whose main goal is to demonstrate the usability of high-contrast imaging equipment on board a stratospheric balloon flight. Funded by CSA-FAST in 2015, a team of five graduate students and four undergraduate interns at Université Laval accomplished the core tasks of the project.

\subsection{Science behind HiCIBaS}

As opposed to Jules Verne's Five weeks in a balloon, HiCIBaS aimed at a one-night mission as part of the Strato-Science 2018 Campaign [4]. After two years of hard work, the HiCIBaS payload 
launched from Timmins Stratospheric Balloon Base on August $25^{\text {th }}, 2018$ at 11:18 pm. The flight onboard the CNES gondola CARMEN provided $6.8 \mathrm{~h}$ of nighttime flight at an altitude of approximately $37 \mathrm{~km}$. HiCIBaS was one of the seven experiments included in that flight.
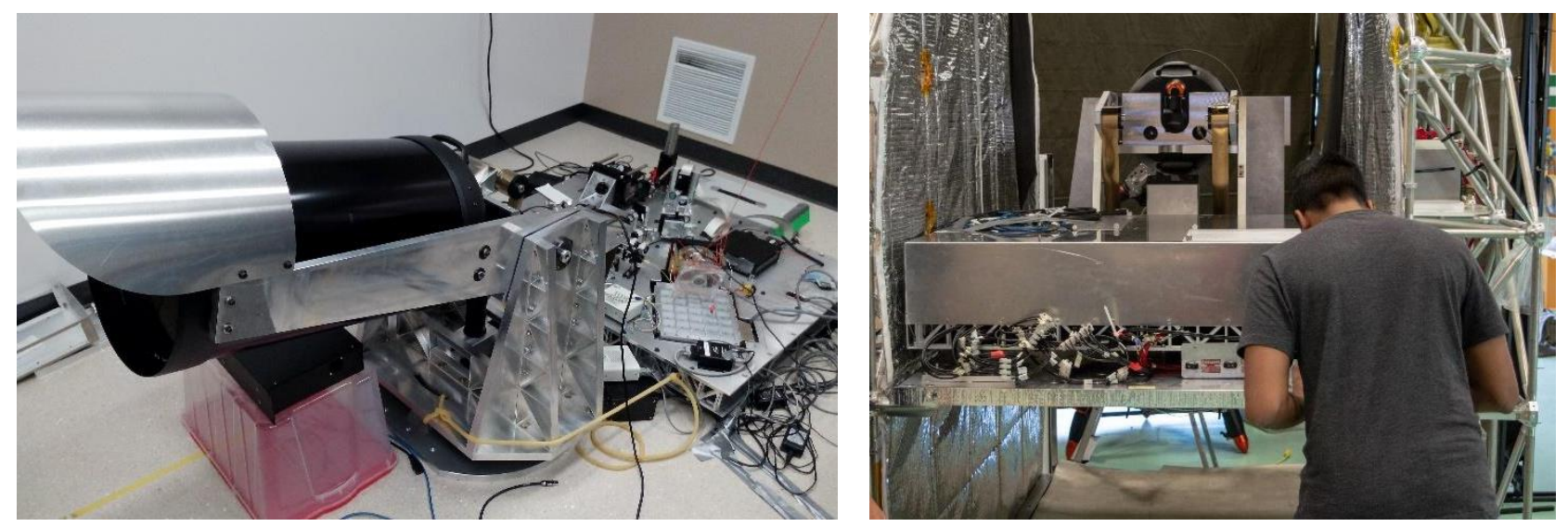

Figure 1. HiCIBaS payload in the laboratory (left) and integrated in the gondola (right)

$\mathrm{HiCIBaS}$ is a research and development project that has five main scientific goals [5]:

1. Develop and test a new type of Low-Order WaveFront Sensor (LOWFS)

2. Develop and test a sub-milli-arcsecond-level pointing system

3. Gather data on and study high-altitude wavefront instabilities and errors

4. Test optical components (DM, Coronagraph) for future high contrast imaging missions

5. Fly the technology in space-like conditions

The complete system architecture and details can be seen at Figure 2. Although every scientific objective wasn't fully achieved at the end of the mission, a significant amount of the development work and on-ground testing required for all the systems was completed. Further to this, critical knowledge for the design of many of the systems was acquired towards the end of the mission that can, undoubtedly, be carried over to future designs of similar payloads. HiCIBaS' project is high-risk by nature. That is why the payload underwent several testing phases prior to the launch, including environmental and on-sky testing, The LOWFS was also tested at Observatoire du Mont-Mégantic for several nights [6]. 


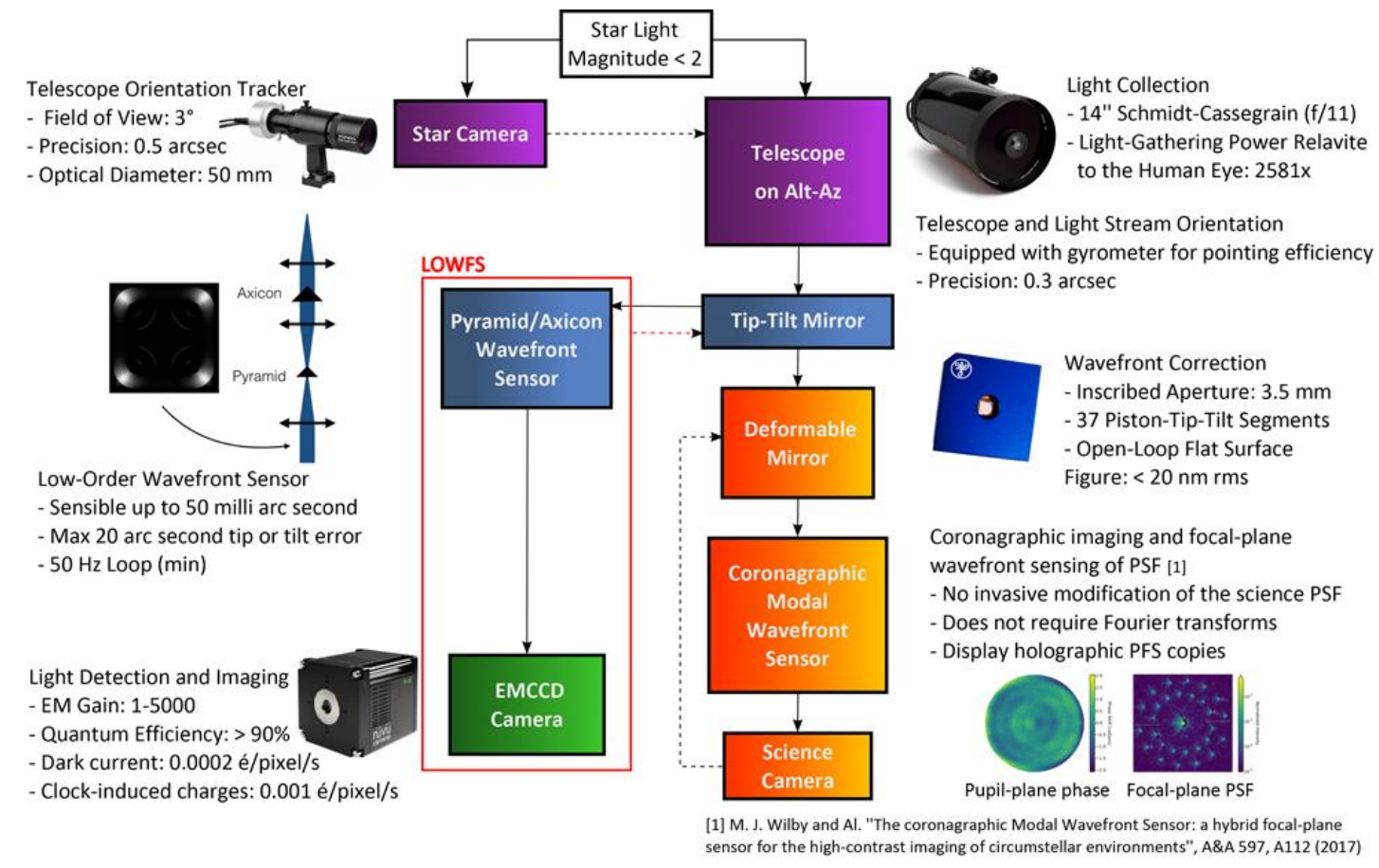

Figure 2. HiCIBaS systems schematic of the complete mission concept including the telescope and star tracker, the LowOrder WaveFront Sensor (LOWFS) and the DM arm that includes the deformable mirror, coronagraph and science camera [7].
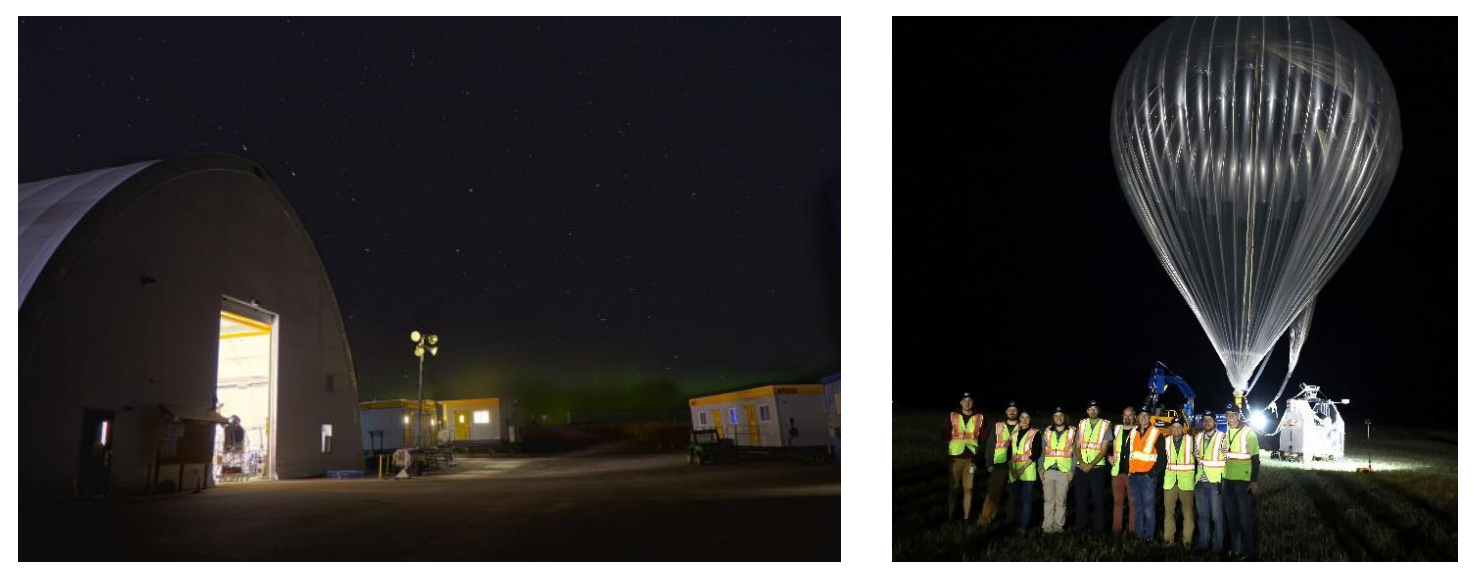

Figure 3. HiCIBaS payload was tested several times before flight, including at the launch base (left). HiCIBaS' Canadian and Dutch teams in front of the gondola with auxiliary balloon on the runway right before launch (right).

\subsection{An industrial and international collaboration for the benefits of trainees}

As part of its payload, HiCIBaS used two EMCCD cameras from a Canada-based company [7]. Not only did the mission allow to test instruments in space-like conditions, but it also provided fruitful interaction between trainees and industry professionals. Other industrial collaborations for specific components and tasks of the mission, such as the deformable mirror used in the high-contrast imaging 
arm, optomechanical design, and panoramic imaging to capture the launch, contributed to a rich learning environment for students.

HiCIBaS has also presented an opportunity for international collaborations with universities and organizations. The most significant is with the Netherlands (Universiteit Leiden, University of Groningen and SRON-Netherlands Institute for Space Research). In addition to an exchange of material (including a coronagraph for high-contrast imaging) and expertise, Dutch and Canadian students had the opportunity to visit the other institution's facilities for internships. Three Dutch students and a researcher also joined the Canadian team on the base before the launch, which was an opportunity for these students to get hands-on experience and to witness the launch of the project.

\section{TRAINING PLAN AND OUTCOMES}

When applying for the CSA-FAST funding opportunity, the applicant is required to submit an exhaustive training plan. The proposed training plan included a research plan for each student, the possibility for co-supervision between PIs of the project depending on the research topic, recommended courses, participation in regular meetings with collaborators, relevant conferences to attend, annual information evaluation, and potential industrial or international internships. While the assessment of the quality of the training is not a current requirement for FAST recipients, we decided to conduct a formal survey among the project's five Université Laval graduate students to evaluate the efficiency of the training plan, and to verify if the HiCIBaS project was able to meet FAST training requirements.

\subsection{HiCIBaS in relation to FAST, CSA, CNES and STRATOS}

When asked about which FAST objective (see section 2 for complete list) has been the most successfully accomplished by HiCIBaS project, students identified 'provide students with opportunities to participate in hands-on activities to gain knowledge and acquire the skills sought by employers in the space sector'. The launch has been identified has the ultimate highlight of the experience, and strongly contributed to the hands-on experience. The support of CSA and CNES personnel, and the STRATOS program and launch base were also mentioned as factors enhancing the learning and scientific experience.

\subsection{Related training opportunities}

Students were asked to identify the training opportunities that were the most useful to them outside of the university. Participation in international conferences and summer schools were the most relevant experiences according to the team. Some of the students mentioned that training opportunities available earlier in the process would have guided their future work and helped them significantly. It is important to note that the international internships took place after the survey.

\subsection{Acquiring new skills for the next step}

Students were also asked to evaluate their impression on specific statements related to technical expertise, soft skills and industry, with respect to their status before starting the HiCIBaS project. 
Results are shown at Fig. 4. Students feel they are fully ready to enter the workforce or pursue another degree in graduate school. They also consider that they significantly improved their project management skills, leadership and communication skills.

Surprisingly, with all the technical work accomplished and hands-on experience, their confidence in their technical skills is among the lowest ranking in the survey. When asked if the mission succeeded in reaching all of the scientific goals outlined in section 3.1, the students mentioned that three objectives were fully completed, one was partially completed, and one was not completed. This perception of accomplishment of scientific objectives could explain why 'I master the technical aspects I worked on' and 'I trust my technical knowledge' have among the lowest scores in Fig. 4.

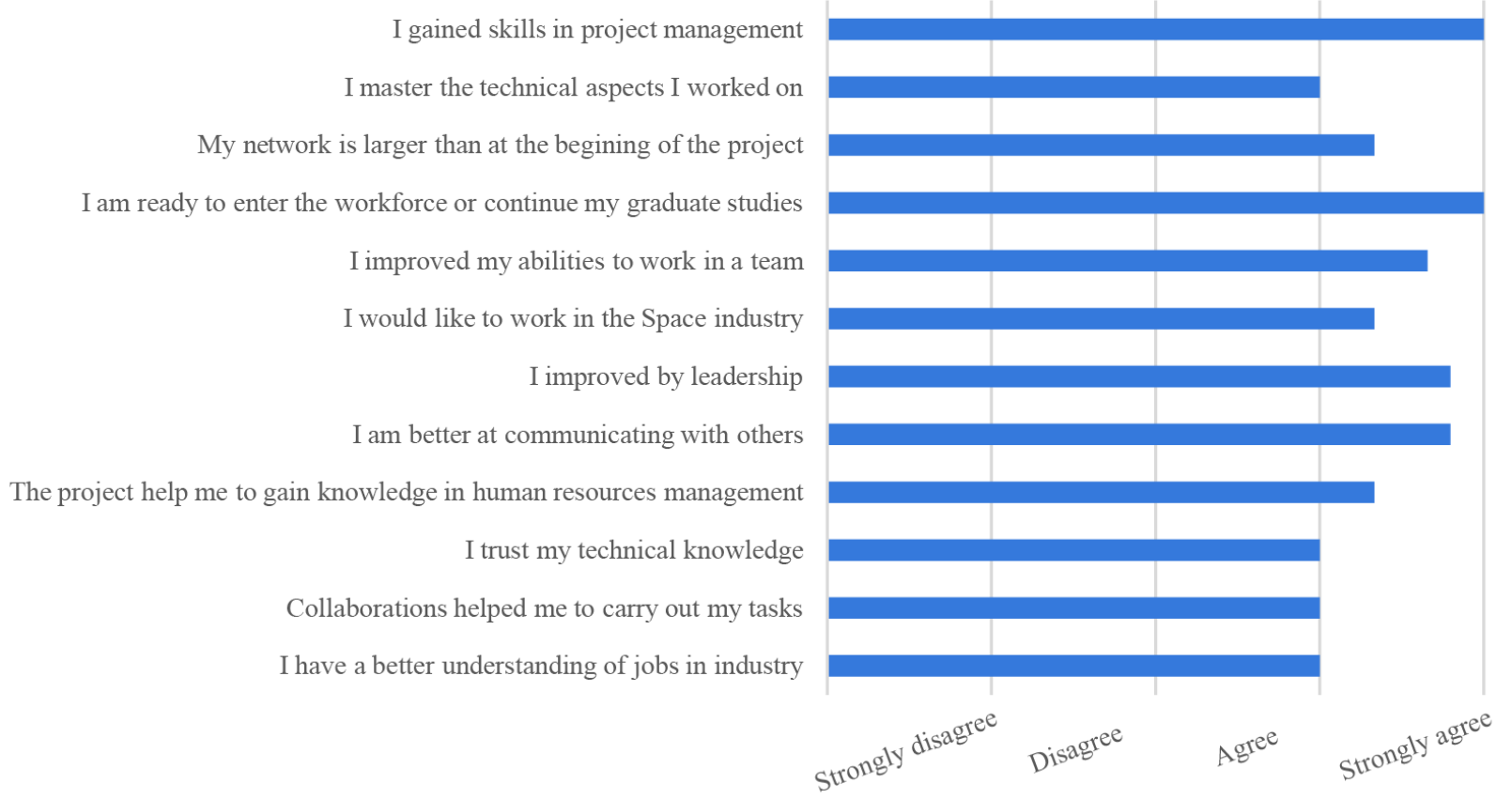

Figure 4. Students were asked to rate various claims with respect to their abilities before the beginning of the project

\section{AN OPPORTUNITY TO REACH OUT TO FAMILY, FRIENDS, AND MORE}

Research not only provides a great training opportunity, it also allows to get in touch with the public and young people. It is not always an easy task. Research material, such as texts and graphics, is often not appropriate for the general public. However, the team felt that, considering the attractive longterm objective of exoplanet identification, the various fields of science and engineering addressed the project, the fun, and dynamic personality of the team, and the industry and international collaboration components, HiCIBaS was a great project to reach out to the public, both locally and internationally. We decided to invest efforts in selected areas to share the research and training experience and the science with a broader audience. 
We built a website with minimal information, and updated it monthly with news. We created a Facebook page and made regular publications in various forms: videos, graphics, student profiles, related news from the science world, pictures, updates on the project, information about partners, etc. By linking our content to other pages from partners and collaborators, we were able to reach more people and get more than 200 likes in the month prior to the launch. The material available from the Canadian Space Agency also made it easier for us to produce or adapt content. We also live-streamed the launch using our Facebook page. We used our network to enhance the visibility of the project among various audiences with articles in professional societies' journal and Facebook page, online medias such as Futura-Science, local newspapers, SETI Institute website, local research centre websites and university news. We also worked with the university's communications services and CSA to increase the efficiency of our outreach efforts.

\section{CONCLUSION}

The stringent and result-oriented training requirements of the Canadian Space Agency FAST funding activity ensure that research funding is used to further knowledge, and also to train the next generation of scientists and engineers. The HiCIBaS project, funded by FAST in 2015, is a great example of how industry, universities and funding agencies can work together to provide a relevant hands-on educational experience for students and train tomorrow's workforce. The retrospective survey completed after the project by the five graduate students who led the project at Université Laval showed that not only did the project meet the FAST requirements in terms of training, but that the students felt they were ready for the next step in their career.

\section{REFERENCES}

[1] J. Burchill, A. Higgins, D. Hudak, A. Moffat, B. Natterfield, K. A. Walker, J. Halliwell and L. Beauchamp, "2010 Workshop on Suborbital Platforms and Nanosatellites Report," Canadian Space Agency, St-Hubert, 2010.

[2] CSA, "About the FAST funding initiative," 1403 2019. [Online]. Available: http://www.asccsa.gc.ca/eng/funding-programs/programs/fast/about.asp. [Accessed 2204 2019].

[3] HiCIBaS, "HiCIBaS Balloon-borne mission," Université Laval, 0110 2018. [Online]. Available: http://hicibas.copl.ulaval.ca/. [Accessed 2204 2019].

[4] CSA, "Strato-Science 2018 campaign," 0811 2018. [Online]. Available: http://www.asccsa.gc.ca/eng/sciences/balloons/campaign-2018.asp. [Accessed 2204 2019].

[5] O. Côté, G. Allain, D. Brousseau, M.-P. Lord, S. Ouahbi, M. Ouellet, D. Patel, S. Thibault, C. Vallée, R. Belikov, E. Bendek, C. Blain, C. Bradley, O. Daigle, C. de Jong, D. Doelman, R. Doyon, F. Grandmont, M. Helmbrecht, M. Kenworthy, D. Lafrenière, F. Marchis, C. Marois, S. Montminy, F. Snik, G. Vasisht, J.-P. Véran and P. Vincent, "A precursor mission to high contrast imaging balloon system," in Proc. SPIE 10702, Ground-based and Airborne Instrumentation for Astronomy VII; $1070248,2018$. 
[6] G. Allain, D. Brousseau, S. Thibault, C. Vallée, M. Ouellet, J.-P. Véran and O. Daigle, "First onsky results, performance, and future of the HiCIBaS-LOWFS," in Proc. SPIE. 10703, Adaptive Optics Systems VI; 107035T, 2018.

[7] O. Daigle, J. Turcotte, O. Djazovski, É. Gloutnay, F. Grandmont, J. Veilleux, D. Patel and S. Thibault, "TRL-5 EMCCD controller for space applications," in Proc. SPIE 10698, Space Telescopes and Instrumentation 2018: Optical, Infrared, and Millimeter Wave; 106986E, 2018.

\section{Acknowledgments}

The HiCIBaS project was supported by the Canadian Space Agency. The authors would like to thank Martin Lebeuf for helping us to understand the context that led to the creation of the CSA-FAST funding opportunity. 